\title{
Classical Photoreceptors Regulate Melanopsin mRNA Levels in the Rat Retina
}

\author{
Katsuhiko Sakamoto, Cuimei Liu, and Gianluca Tosini \\ Neuroscience Institute and National Science Foundation Center for Behavioral Neuroscience, Morehouse School of Medicine, Atlanta, Georgia $30310-1495$
}

Recent studies have demonstrated that melanopsin is a key photopigment in the mammalian circadian system. This novel opsin is exclusively expressed in retinal ganglion cells that are intrinsically sensitive to light, perhaps responding via a melanopsin-based signaling pathway. Previous investigations using transgenic mice have also demonstrated that ablation of the classical photoreceptors and of melanopsin prevents entrainment of several circadian rhythms, thus demonstrating that these photoreceptors are necessary and sufficient for circadian photoreception. In this study, we investigated the effect of photoreceptor degeneration on melanopsin mRNA regulation in RCS/N-rdy rats (Royal College of Surgeons rats with a defect in the retinal dystrophy gene). We used animals at postnatal day 21 (P21), P33, P45, and P60. At P60 degeneration of the retina in RCS/N-rdy has advanced to the point where the majority of the photoreceptors have degenerated. Our data indicate that melanopsin mRNA levels were rhythmic in light/dark cycle and in constant darkness in congenic controls (RCS/N-rdy ${ }^{+}$) and in RCS/N-rdy at P21 (i.e., before the degeneration of the photoreceptors). On the other hand, in $\mathrm{RCS} / \mathrm{N}-r d y$ at P60, melanopsin mRNA levels were greatly reduced $(<90 \%)$ and not rhythmic. Photoreceptor degeneration did not affect the expression of pituitary adenylate cyclase-activating polypeptide mRNA (a marker for melanopsin-containing ganglion cells). Our results suggest that classical photoreceptors (rods and cones) regulate the expression of melanopsin mRNA in the rat. Because RCS/N-rdy rats are a model for studies on retinitis pigmentosa in human, our data may provide an important insight on melanopsin function in patients affected by retinitis pigmentosa.

Key words: melanopsin; circadian rhythm; RCS rat; circadian photoreception; retina; photoreceptor degeneration

\section{Introduction}

The study of the photoreceptors and photopigments that mediate circadian photoreception in mammals is emerging as a new and exciting aspect of retinal neurobiology. A series of elegant studies in the 1990s demonstrated that classical photoreceptors (rods and cones) are not necessary for the entrainment of circadian rhythms in mammals (Foster et al., 1991; Freedman et al., 1999; Lucas et al., 1999), thus indicating that an undiscovered photoreceptor (photopigment) in the mammalian retina is responsible for the photoentrainment of circadian rhythms.

The most likely candidate to emerge as the circadian retinal photopigment is a mammalian homolog of Xenopus melanopsin (Provencio et al., 1998). In mammals, melanopsin mRNA (and protein) is only expressed in a small population of retinal ganglion cells (RGCs) (Provencio et al., 2000) that are directly photosensitive (Berson et al., 2002), expresses pituitary adenylate cyclase-activating polypeptide (PACAP) (Hannibal et al., 2002), and gives origin to the retinohypothalamic tract (Gooley et al., 2001; Hattar et al., 2002). Additional studies have shown that

Received June 28, 2004; revised Sept. 3, 2004; accepted Sept. 22, 2004.

This work was supported by National Institutes of Health Grant NS 43459 and National Science Foundation Center for Behavioral Neuroscience Grant IBN-9876754 (G.T.). We thank Dr. Jens Hannibal (University of Copenhagen, Copenhagen, Denmark), who kindly provided the melanopsin antiserum.

Correspondence should be addressed to Dr. Gianluca Tosini, Neuroscience Institute, Morehouse School of Medicine, 720 Westview Drive Southwest, Atlanta, GA 30310-1495. E-mail: tosinig@msm.edu.

D01:10.1523/JNEUROSCI.2556-04.2004

Copyright $\odot 2004$ Society for Neuroscience $\quad$ 0270-6474/04/249693-05\$15.00/0 melanopsin (or Opn4) plays an important role in the complex system that regulates circadian photoreception (Panda et al., 2002; Ruby et al., 2002; Hattar et al., 2003), sleep-wake states (Gooley et al., 2003), and the pupillary light reflex (Lucas et al., 2003).

New experimental evidence has demonstrated that melanopsinexpressing RGCs may receive input from rod and/or cone photoreceptors (Belenky et al., 2003), thus suggesting that some interaction may exist between the classical photoreceptors and this newly discovered photoreceptive system. However, a previous study has reported that, in mice lacking rods and cones, melanopsin regulation was not affected by the loss of the photoreceptors (Semo et al., 2003).

Retinitis pigmentosa (RP) is one of the leading causes of blindness in humans (Kaplan et al., 1990). Although RP is most commonly caused by defects intrinsic to photoreceptors, several cases have been identified with recessive mutations of the receptor tyrosine kinase Mertk gene (Li et al., 2000). A recent paper has reported that the gene encoding for Mertk corresponds to the retinal dystrophy $(r d y)$ locus of the RCS (Royal College of Surgeons) rat (D'Cruz et al., 2000). In this animal, which is the most studied of the available animal models of retinal dystrophy, the gene defect renders cells of the retinal pigment epithelium unable to phagocytose and shed photoreceptor outer segments at a normal rate (Dowling and Sidman, 1962). This inability leads to photoreceptor cell death.

Therefore, RCS/N-rdy rats represent a useful model to investigate the effect of photoreceptor degeneration on melanopsin 
expression and to understand the possible function(s) of melanopsin in humans affected by RP.

The aim of this study was to investigate the pattern of expression of melanopsin mRNA under different photic conditions and the effect that photoreceptor degeneration has on melanopsin mRNA regulation in $\mathrm{RCS} / \mathrm{N}-r d y$ rats. Our data indicate that photoreceptor degeneration induced the loss of photic and circadian control of melanopsin mRNA, and melanopsin transcripts were dramatically reduced in retinas lacking photoreceptor cells. The reduction in melanopsin transcripts is not attributable to the loss (death) of melanopsin-positive RCGs but rather to a downregulation of melanopsin mRNA transcription within these cells. These data indicated that classical photoreceptors are required for melanopsin mRNA expression.

\section{Materials and Methods}

Animals and tissue collection. Tan-hooded RCS/N- $r d y^{+}$rats homozygous for the normal $r d y$ allele and congenic age-matched RCS/N-rdy homozygotes with retinal dystrophy were used in this study. Animals were raised from birth at Morehouse School of Medicine in a $12 \mathrm{hr}$ light/dark (LD) cycle of illumination with lights (250 lux) on from Zeitgeber time 0 (ZT0) to ZT12. Food and water were available ad libitum. We used animals at postnatal day 21 (P21), P33, P45, and P60. At P60, degeneration of the retina in $\mathrm{RCS} / \mathrm{N}-r d y$ has advanced to the point at which the majority of the photoreceptors have degenerated (Dowling and Sidman, 1962; Doyle et al., 2002).

To investigate the expression of melanopsin in LD cycles, animals were killed at ZT2, ZT6, ZT10, ZT14, ZT18, and ZT22. When animals were killed during the night, the procedure was performed in dim red light $(<$ 1 lux). To investigate the pattern of expression in constant darkness (DD), rats were transferred into constant darkness for $2 \mathrm{~d}$ before being killed. Samples were then collected on the third day at circadian time 2 (CT2), CT6, CT10, CT14, CT18, and CT22. Retinas were dissected, immediately frozen on dry ice, and stored at $-80^{\circ} \mathrm{C}$.

All procedures were approved by the Animal Care and Use Committee and conformed to National Institutes of Health guidelines.

Real-time quantitative reverse transcription-PCR. Total RNA was isolated form each retina using TRIZOL reagent (Invitrogen, Grand Island, $\mathrm{NY}$ ) after sonication. RNA was treated with DNase I to remove any traces of genomic DNA. First-strand cDNA was synthesized from $1 \mu \mathrm{g}$ of each RNA sample using oligo-dT and Omniscript reverse transcriptase (Qiagen, Valencia, CA) according to the protocol of the manufacturer. Each set of samples was simultaneously processed for RNA extraction, DNase I treatment, cDNA synthesis, and PCR. Real-time quantitative reverse transcription (RT)-PCR was performed with SYBR Green using $i$ Cycler (Bio-Rad, Hercules, CA). Briefly, primers used were as follows: for rat melanopsin (GenBank accession number AY072689), forward, 5'atctggtgatcacacgtcca-3' and reverse, 5' -tagtcccaggagcaggatgt-3' (169 bp product, spanning a $\sim 600 \mathrm{bp}$ intron); for rat PACAP (GenBank accession number M63006), forward, 5' -acagcgtctcctgttcacct- $3^{\prime}$ and reverse, $5^{\prime}$-cctgtcggctgggtagtaaa-3' (179 bp product, spanning a $\sim 2000$ bp intron); for rat rhodopsin (GenBank accession number Z46957), forward, $5^{\prime}$-gcagtgttcatgtgggattg- $3^{\prime}$ and reverse, 5'-ctgccttctgagtggtagcc-3' (191 bp product); for rat blue cone opsin (GenBank accession number AF051163), forward, 5'-gtaccacattgctcccgtct-3' and reverse, 5' agacctgctacagagcccaa- $3^{\prime}$ (282 bp product); for rat green cone opsin (GenBank accession number AF031528), forward, $5^{\prime}$-tcatttcctgggagagatgg- $3^{\prime}$ and reverse, $5^{\prime}$-tttctgttgctttgccactg-3' (326 bp product); and for rat Gapdh (glyceraldehyde-3-phosphate dehydrogenase) (GenBank accession number X02231), forward, $5^{\prime}$-agacagccgcatcttcttgt- $3^{\prime}$ and reverse, $5^{\prime}$-tgatggcaacaatgtccact- $3^{\prime}$ (142 bp product). Cycling parameters were $95^{\circ} \mathrm{C}$ for $8 \mathrm{~min}$ from activate TaqDNA polymerase, 40 cycles of $95^{\circ} \mathrm{C}$ for $15 \mathrm{sec}, 60^{\circ} \mathrm{C}$ for $45 \mathrm{sec}$, and $72^{\circ} \mathrm{C}$ for $30 \mathrm{sec}$. The fluorescence of the accumulating product was measured after each cycle at $88^{\circ} \mathrm{C}, 89^{\circ} \mathrm{C}, 86^{\circ} \mathrm{C}$, $86^{\circ} \mathrm{C}, 87^{\circ} \mathrm{C}$, and $85^{\circ} \mathrm{C}$ (i.e., $4^{\circ} \mathrm{C}$ below the product temperature) for melanopsin, PACAP, rhodopsin, blue cone opsin, green cone opsin, and Gapdh mRNA, respectively. To confirm the specificity of PCR products, melting curves were determined using $i$ Cycler software, and samples were run on an agarose gel. The amount of amplified product was calculated from plasmid standards. Expression levels of each transcript were normalized by comparison with the amount of Gapdh mRNA

Immunohistochemistry and Western blot. Eyeballs obtained from RCS/ $\mathrm{N}-r d y^{+}$and RCS/N-rdy animals at P60 killed at ZT14 were punctured and then fixed with $4 \%$ paraformaldehyde in PBS, pH 7.0, for $6 \mathrm{hr}$ at $4^{\circ} \mathrm{C}$. The eyes were transferred to a solution of $30 \%$ sucrose for $12-14 \mathrm{hr}$, embedded in Tissue-Tek OCT compound (Miles, Elkhart, IN), cut into 12- to $16-\mu \mathrm{m}$-thick cryosections, and stored at $-80^{\circ} \mathrm{C}$ until processed for immunohistochemistry using a procedure described by Hannibal et al. (2002). A well characterized rabbit anti-melanopsin antiserum (code 41 K9; generous gift from Dr. Jens Hannibal, University of Copenhagen, Copenhagen, Denmark) diluted 1:20,000 combined with tyramide amplication was used. Slides were mounted and then viewed with a Zeiss (Oberkochen, Germany) Axioskop microscope equipped with epifluorescence. The Western blot was performed as described by Hannibal et al. (2002) using the same antibody (code 41K9) diluted 1:1000. Both the immunohistochemistry and Western blot were repeated at least three times with independent samples.

\section{Results}

\section{Effect of photoreceptor degeneration on} melanopsin regulation

To investigate the effects of photoreceptor degeneration on melanopsin, we measured melanopsin mRNA levels in RCS rats at P60 (Fig. 1A). In RCS/N-rdy (controls), melanopsin transcripts showed a high-amplitude rhythm in LD and in DD (ANOVA; $p<0.001$, in both cases), with high levels during early night (ZT10-ZT14) relative to the early day (ZT22-ZT2). Thus, we can conclude that, in RCS/N-rdy ${ }^{+}$(controls), melanopsin mRNA expression is controlled by the circadian clock. Surprisingly, in animals lacking photoreceptors (RCS/N-rdy), melanopsin mRNA levels were significantly reduced $(\sim 90 \%)$, and no rhythmic expression was observed (ANOVA; $p>0.33$ ) (Fig. 1A).

Melanopsin-immunoreactive cells were observed in the ganglion cell layer of RCS/N-rdy ${ }^{+}$(Fig. $1 B$ ) but not in RCS/N-rdy (Fig. 1C). The distribution pattern of melanopsin immunoreactivity in RCS/N-rdy ${ }^{+}$animals was similar to that described in previous studies (Hannibal et al., 2002; Hattar et al., 2002). In addition to immunohistochemistry, expression of melanopsin protein was confirmed by Western blotting in RCS/N-rdy ${ }^{+}$but not in RCS/N-rdy rat (Fig. 1D). In RCS/N-rdy ${ }^{+}$melanopsin seems to be more abundant at ZT14 than at ZT2 (Fig. 1D).

\section{The mutation in the Mertk gene is not directly responsible for the results obtained in $\mathrm{RCS} / \mathrm{N}-r d y$}

To test whether the change in melanopsin mRNA expression was a consequence of the mutation in the Mertk gene or the result of photoreceptor degeneration, we investigated the pattern of expression of melanopsin mRNA in RCS/N-rdy rats at P21 (i.e., before the photoreceptors start to degenerate). Melanopsin transcripts were clearly rhythmic in RCS/N-rdy rats at P21 (ANOVA; $p<0.01$ ) (Fig. 2), with overall levels that were not significantly different from what was observed in RCS/N-rdy ${ }^{+}$of the same age ( $t$ tests; $p>0.1$ in all cases) (Fig. 2). The pattern of expression of melanopsin mRNA was also similar among RCS/N-rdy at P21 and RCS/N-rdy ${ }^{+}$at P21 and P60 (Figs. 1A, 2).

\section{PACAP mRNA is not affected by photoreceptor degeneration}

Previous studies have shown that, although RCS/N-rdy mutants undergo a process that leads to the loss of the photoreceptors, the RGCs are not affected, and, indeed, we found that Thy- 1 mRNA levels (a marker for ganglion cells) were not significantly different between $\mathrm{RCS} / \mathrm{N}-r d y$ and $\mathrm{RCS} / \mathrm{N}-r d y^{+}$rats at $\mathrm{P} 60$ ( $t$ test; $p>0.1$ ) 

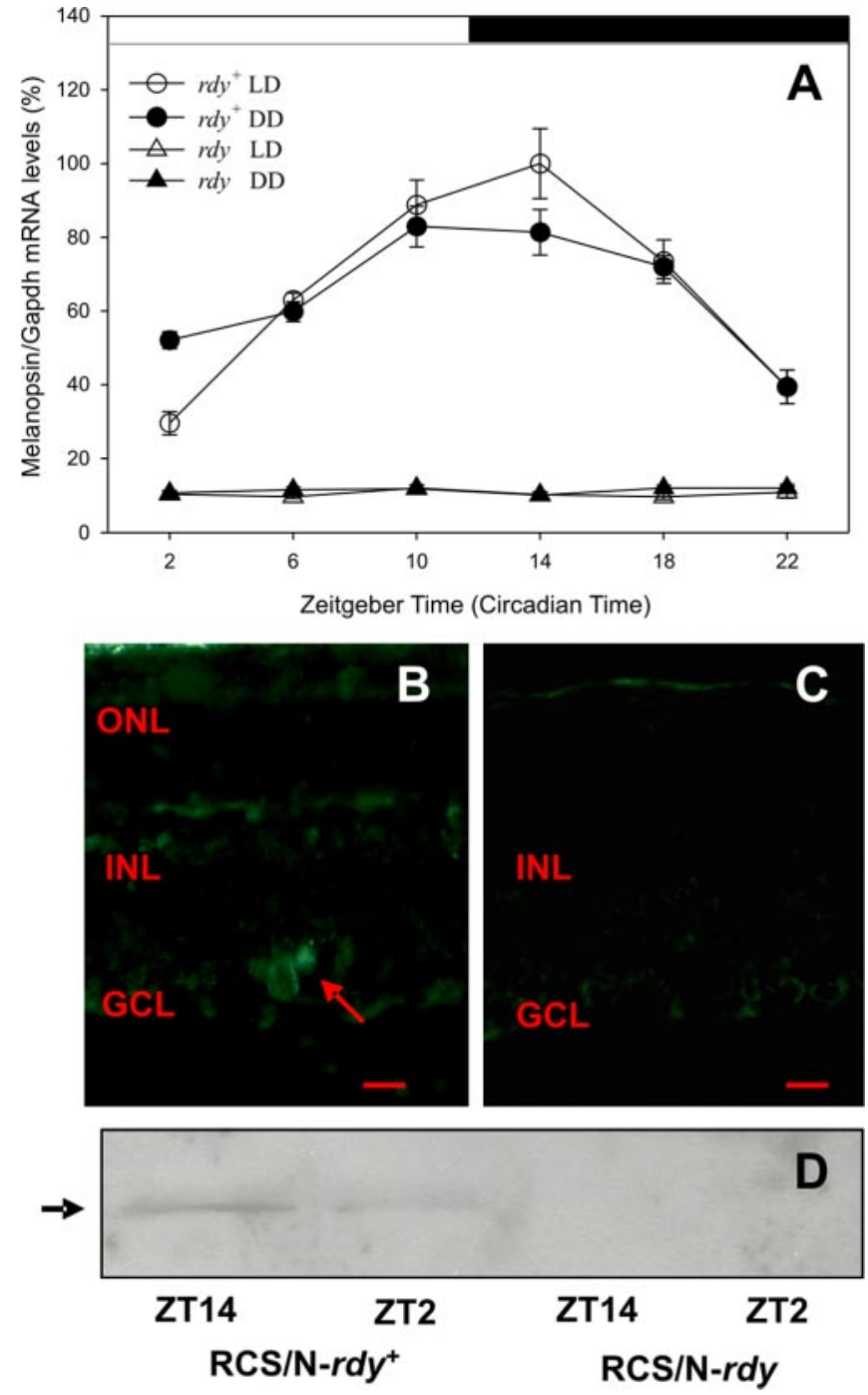

Figure 1. A, Melanopsin mRNA levels in $\mathrm{RCS} / \mathrm{N}-r d y$ and $\mathrm{RCS} / \mathrm{N}-r d y{ }^{+}$retina at $\mathrm{P} 60$. Melanopsin mRNA levels were measured using real-time quantitative RT-PCR. mRNA levels were rhythmic in LD and DD (ANOVA; $p<0.001$ ) in RCS/N-rdy ${ }^{+}$animals but not in RCS/N-rdy (ANOVA; $p>0.1$ ). Data are expressed as mean $\pm S E M(n \geq 4$ retinas). All values were normalized with respect to the values obtained in $\mathrm{RCS} / \mathrm{N}-\mathrm{rdy}^{+}$animals at ZT14. The white bar at the top of the graph represents the period of light (ZTO-ZT12), and the black bar represents the period of darkness (ZT12-ZT24) when the rats were exposed to LD cycles. Photomicrographs showing the expression pattern of melanopsin-immunoreactive cells (arrow) in a 60-d-old RCS/N-rdy ${ }^{+}$rat with a normal retina ( $B$ ) and in a 60-d-old RCS/N-rdy ( (). Scale bars, $20 \mu \mathrm{m}$. ONL, Outer nuclear layer; INL, inner nuclear layer; $\mathrm{GCL}$, ganglion cell layer. In the dystrophic retina, the photoreceptor layer is absent. D, Western blot of protein extracts from pooled retinas probed with the melanopsin antibody. Immunoreactivity (as a $50 \mathrm{kDa}$ band, arrow) was present in $\mathrm{RCS} / \mathrm{N}-\mathrm{rdy}{ }^{+}$ at ZT14, and a weaker band was also present at ZT2. No immunoreactivity was detected in $\mathrm{RCS} / \mathrm{N}-$ rdy rats.

(data not shown). However, it must be noted that melanopsin is only expressed in a small subpopulation (1-2\%) of RGCs, and, therefore, small changes in Thy-1 mRNA levels that may result as a consequence of the loss of a few melanopsin-positive RGCs may be difficult to detect. A previous study has reported that melanopsin mRNA and PACAP mRNA are coexpressed in the same RGCs. Therefore, we decided to investigate the effects of photoreceptor degeneration on the levels of PACAP mRNA in RCS/N$r d y$ to test whether the observed changes in melanopsin mRNA were attributable to the loss (death) of a subpopulation of RGCs or to a change in the regulation of melanopsin transcription

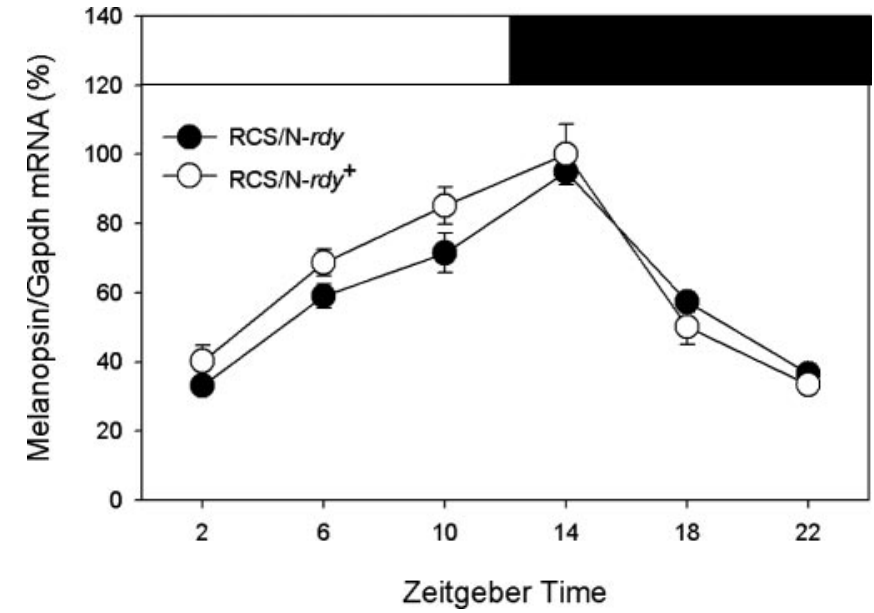

Figure 2. Melanopsin mRNA levels in RCS/N-rdy at P21. All values were normalized with respect to the values obtained at $Z T 14$ in $\mathrm{RCS} / \mathrm{N}-r d y^{+}$. The white bar at the top of the graph represents the period of light (ZTO-ZT12), and the black bar represents the period of darkness (ZT12-ZT24) when the rats were exposed to LD cycles. Data are expressed as mean \pm SEM ( $n$ $\geq 4$ retinas).

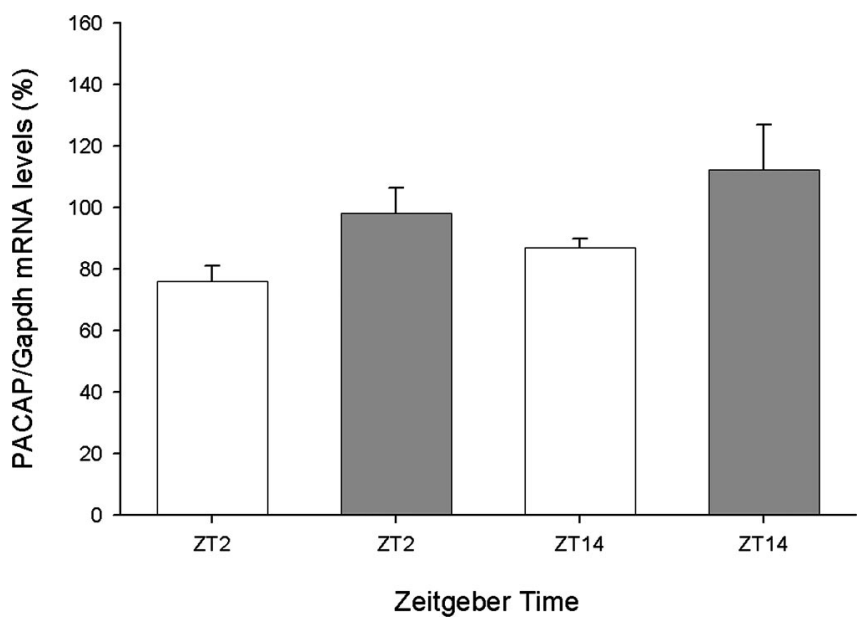

Figure 3. $\quad P A C A P m R N A$ levels in $\mathrm{RCS} / \mathrm{N}-r d y^{+}$(white bars) and RCS/N-rdy (black bars) retinas at $P 60$ at $Z$ T2 and ZT14. Data are expressed as mean \pm SEM ( $n \geq 4$ retinas). PACAP mRNA levels did not differ between RCS/N-rdy ${ }^{+}$and RCS/N-rdy at both time points ( $p>0.05$ in all cases).

within these cells. Surprisingly, we observed that PACAP transcripts were not reduced by the loss of photoreceptors ( $t$ tests; $p>$ 0.1) (Fig. 3), thus demonstrating that the observed reduction in melanopsin mRNA is not attributable to the loss of melanopsinpositive RGCs but rather to the change in the synthesis of this putative photopigment that follows the degeneration of the photoreceptor cells.

Photoreceptor loss affects melanopsin mRNA levels

In our last experiment, we investigated the effect of photoreceptor degeneration on the levels and pattern of expression of melanopsin mRNA in RCS-rdy rats at P21, P33, P45, and P60. Melanopsin mRNA levels showed a steady decrease between P21 and P60 ( $t$ tests; $p<0.01$ in all cases) (Fig. $4 A$ ). As expected, rhodopsin transcripts showed a dramatic reduction with the progression of photoreceptor loss (Fig. $4 \mathrm{~B}$ ). The greatest reduction in the level of rhodopsin mRNA was observed between P33 and P45. Green cone opsin (Fig. 4C) and blue cone opsin (Fig. 4D) transcripts showed a significant decrease between P21 and P60 ( $t$ test; $p<$ 

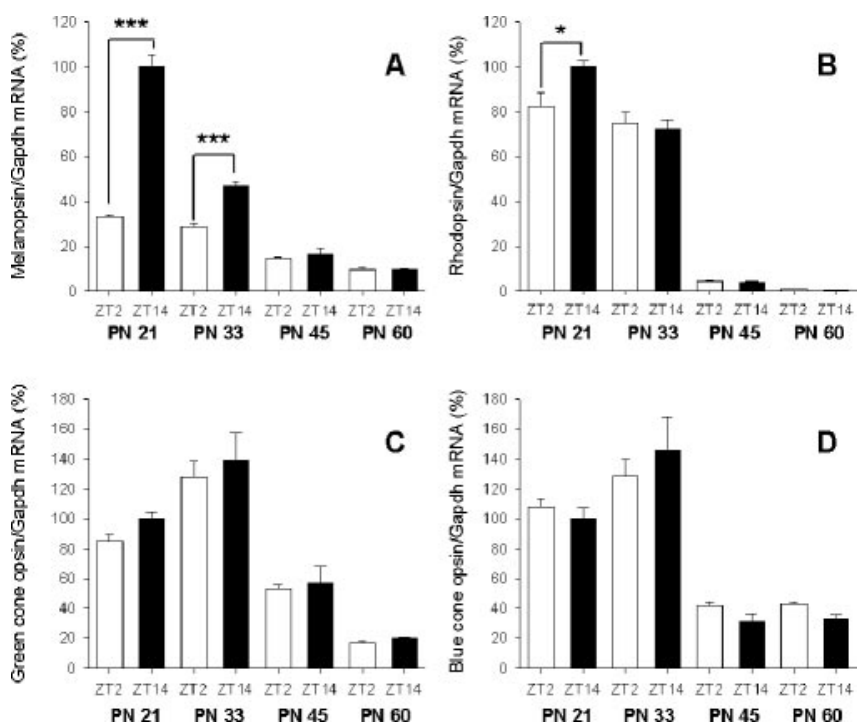

Figure 4. Changes in photopigments $m R N A$ in $R C S / N-r d y$ rats at $\mathrm{P} 21, \mathrm{P} 33, \mathrm{P} 45$, and $\mathrm{P} 60 . \mathrm{A}$, Melanopsin; $B$, rhodopsin; $C$, green cone opsin; and D, blue cone opsin. mRNA levels were measured at ZT2 (white bars) and ZT14 (black bars). These time points correspond to the trough and the peak of the rhythm for melanopsin, rhodopsin, and green cone opsin transcripts, whereas blue cone opsin mRNA was found not rhythmic (K. Sakamoto and G. Tosini, unpublished observations). Data are expressed as mean $\pm \operatorname{SEM}\left(n \geq 3\right.$ retinas). ${ }^{*} p<0.05 ;{ }^{* * *} p<0.001$. PN, Postnatal day.

0.01 in all cases), but the reduction was not as dramatic as observed in rhodopsin mRNA (Fig. 4).

Interestingly, melanopsin mRNA rhythmicity disappeared between P33 and P45 (i.e., in parallel with the reduction in rhodop$\sin$ mRNA) (Fig. $4 A, B)$.

\section{Discussion}

The focus of this study was to investigate the effects of photoreceptor degeneration on the regulation of melanopsin mRNA in the rat retina. Our data demonstrated that, in animals lacking photoreceptors, melanopsin mRNA transcription is arrhythmic, and melanopsin protein is not detectable (Fig. $1 A-D$ ). In addition, we also demonstrated that the observed changes in melanopsin mRNA regulation are an outcome of photoreceptor degeneration rather than a direct effect of the Mertk mutation in RCS/ $\mathrm{N}-r d y$ rats (Figs. 2, 4). Finally, our results indicate that the reduction in melanopsin mRNA is not attributable to the loss of RGCs because Thy-1 mRNA and, more importantly, PACAP mRNA levels are not affected by the reduction in the photoreceptor number (Fig. 3).

Although several studies have reported the distribution of melanopsin mRNA and protein (Provencio et al., 2000; Gooley et al., 2001; Hattar et al., 2002), we found that only one paper has suggested a possible day-night difference in melanopsin expression (Hannibal et al., 2002). The data presented here agree with those preliminary results and clearly demonstrate that melanop$\sin$ mRNA levels are rhythmic in LD and DD, with higher levels at ZT (CT) 14 and lowest at ZT (CT) 2. Moreover, our data show that melanopsin transcripts are not affected by illumination once the photoreceptors have degenerated (Figs. 1A, 4). Such a result suggests that melanopsin mRNA levels in the RCS/N-rdy at P60 do not generate enough melanopsin protein that would be required to successfully perform the normal phototransduction processes.

A recent study has reported that the gene encoding for the receptor tyrosine kinase (Mertk) corresponds to the retinal dys- trophy ( $r d y$ ) locus of the RCS rat (D'Cruz et al., 2000). The finding that RCS/N-rdy rats at P2 1 showed a clear rhythm in melanopsin mRNA, which was not different from what was observed in control animals (Fig. 2), together with the fact that melanopsin and Mertk transcripts are not expressed in the same type of retinal cells (D'Cruz et al., 2000; Provencio et al., 2000) demonstrated that the results obtained are not attributable to the direct effect of the mutation in Mertk gene. Hence, we believe the observed results could be an indirect consequence of (or mediated by) photoreceptor degeneration.

Previous investigations have reported that photoreceptor degeneration in RCS/N-rdy rat does not affect the ganglion cell layer (Villegas-Perez et al., 1998). Our data confirm and expand those data in demonstrating that Thy-1 mRNA [a marker for RGCs (Barnstable and Drager, 1984)] and, more importantly, PACAP mRNA levels are not reduced in rats lacking photoreceptors. Because previous studies have shown that PACAP and melanopsin transcripts in the rat retina are coexpressed and have identical distribution (Hannibal et al., 2002), we can conclude that the observed reduction in melanopsin mRNA levels at P60 was not attributable to the loss (death) of melanopsin-positive RCGs but rather to a downregulation of melanopsin mRNA transcription within these cells.

The rat retina contains rod and cone (green and blue) photoreceptors. Rods represent $\sim 99 \%$ of the photoreceptors, and green cones represent $80-85 \%$ of all the cones (Szel and Rohlich, 1992). Our data show that the reduction in rhodopsin mRNA levels progresses with age, and, by $\mathrm{P} 60,<1 \%$ of rhodopsin mRNA is found in RCS/N- $r d y$ retinas (Fig. $4 B$ ). In addition, our results show that melanopsin mRNA became arrhythmic between P33 and $\mathrm{P} 45$, when a dramatic decrease in rhodopsin mRNA was observed but when cone opsin transcripts are still relatively abundant (Fig. 4C,D). Such a result suggests that rod photoreceptors may be the key players in controlling melanopsin mRNA in rat retina.

As we have mentioned previously, in mice lacking rods and cones, melanopsin mRNA levels were not affected by the loss of photoreceptors (Semo et al., 2003), thus showing a dramatic difference from the result obtained in our study. We believe that there are at least two possible explanations for such a discrepancy. First, it is plausible that the difference in the results obtained between the two studies is attributable to the fact that two different species (rat and mouse) were used. Alternatively, the observed discrepancy can be attributed to the fact that the different mechanisms that lead to photoreceptor degeneration in the rodless-coneless mouse and in the RCS/N-rdy rat may affect melanopsin regulation in a different manner. In that regard, it may be significant to mention that, in some blind subjects, with no conscious perception of light, the photic input to the circadian system is preserved (Czeisler et al., 1995; Klerman et al., 2002). Such a result indicates that different modalities leading to the degeneration of the classical photoreceptors may have different effects on the photoreceptive system mediating circadian photoreception.

Although the data presented here do not question the role of melanopsin as an important component in the pathway involved in circadian photoreception (Panda et al., 2002, 2003; Ruby et al., 2002; Hattar et al., 2003), the finding that melanopsin mRNA transcription is greatly affected by the loss of rod and cone photoreceptors indicates that, at least in the rat, melanopsin mRNA regulation is somehow controlled by the classical photoreceptors.

Finally, we want to mention that melanopsin is believed to be involved in the mediation of several aspects of nonvisual photoreception that may affect human health (Gooley et al., 2003). As 
we have mentioned previously, RCS/N-rdy rats represent a well established model to study RP in human, and our new findings suggest that RP may also affect melanopsin-based photoreception and thus the processes that are mediated by this photopigment.

\section{References}

Barnstable CJ, Drager UC (1984) Thy-1 antigen: a ganglion cell specific marker in rodent retina. Neuroscience 11:847-855.

Belenky MA, Smeraski CA, Provencio I, Sollars PJ, Pickard GE (2003) Melanopsin retinal ganglion cells receive bipolar and amacrine cell synapses. J Comp Neurol 460:380-393.

Berson DM, Dunn FA, Takao M (2002) Phototransduction by ganglion cells innervating the circadian pacemaker. Science 295:1070-1073.

Czeisler CA, Shanahan TL, Klerman EB, Martens H, Brotman DJ, Emens JS, Klein T, Rizzo III JF (1995) Suppression of melatonin secretion in some blind patients by exposure to bright light. N Engl J Med 332:6-11.

D'Cruz PM, Yasumura D, Weir J, Matthes MT, Adberrahim H, LaVail MM, Vollrath D (2000) Mutation of the receptor tyrosine kinase gene Mertk in the retinal dystrophic RCS rat. Hum Mol Genet 9:645-651.

Dowling JE, Sidman RL (1962) Inherited retinal dystrophy in the rat. J Cell Biol 14:73-109.

Doyle SE, McIvor WE, Menaker M (2002) Circadian rhythmicity in dopamine content of mammalian retina: role of the photoreceptors. J Neurochem 83:211-219.

Foster RG, Provencio I, Hudson D, Fiske S, De Grip W, Menaker M (1991) Circadian photoreception in the retinally degenerate mouse $(r d / r d)$. J Comp Physiol [A] 169:39-50.

Freedman MS, Lucas RJ, Soni B, Von Schantz M, Munoz M, David-Gray Z, Foster RG (1999) Regulation of mammalian circadian behavior by nonrod, non-cone, ocular photoreceptors. Science 284:502-504.

Gooley JJ, Lu J, Chou TC, Scammell TE, Saper CB (2001) Melanopsin in cells of origin of the retinohypothalamic tract. Nat Neurosci 4:1165.

Gooley JJ, Lu J, Fischer D, Saper CB (2003) A broad role for melanopsin in nonvisual photoreception. J Neurosci 23:7093-7106.

Hannibal J, Hindersson P, Knudsen SM, Georg B, Fahrenkrug J (2002) The photopigment melanopsin is exclusively present in pituitary adenylate cyclase-activating polypeptide-containing retinal ganglion cells of the retinohypothalamic tract. J Neurosci 22:RC191(1-7).

Hattar S, Liao HW, Takao M, Berson DM, Yau KW (2002) Melanopsincontaining retinal ganglion cells: architecture, projections, and intrinsic photosensitivity. Science 295:1065-1070.

Hattar S, Lucas RJ, Mrosovsky N, Thompson S, Douglas RH, Hankins MW, Lem J, Biel M, Hofmann F, Foster RG, Yau KW (2003) Melanopsin and rod-cone photoreceptive systems account for all major accessory visual functions in mice. Nature 424:76-81.

Kaplan J, Bonneau D, Frezal J, Munnich A, Dufier JL (1990) Clinical and genetic heterogeneity in retinitis pigmentosa. Hum Genet 85:635-642.

Klerman EB, Shanahan TL, Brotman DJ, Rimmer DW, Emens JS, Rizz III JF, Czeisler CA (2002) Photic resetting of the human circadian pacemaker in the absence of conscious vision. J Biol Rhythms 17:548-555.

Li Y, Thompson DA, Weir J, Orth U, Jacobson SG, Apfelstedt-Sylla E, Vollrath D (2000) Mutations in MERTK, the human orthologue of the RCS rat retinal dystrophy gene, cause retinitis pigmentosa. Nat Genet 26:270-271.

Lucas RJ, Freedman MS, Munoz M, Garcia-Fernandez JM, Foster RG (1999) Regulation of the mammalian pineal by non-rod, non-cone, ocular photoreceptors. Science 284:505-507.

Lucas RJ, Hattar S, Takao M, Berson DM, Foster RG, Yau KW (2003) Diminished pupillary light reflex at high irradiances in melanopsinknockout mice. Science 299:245-247.

Panda S, Sato TK, Castrucci AM, Rollag MD, DeGrip WJ, Hogenesch JB, Provencio I, Kay SA (2002) Melanopsin (Opn4) requirement for normal light-induced circadian phase shifting. Science 298:2213-2216.

Panda S, Provencio I, Tu DC, Pires SS, Rollag MD, Castrucci AM, Pletcher MT, Sato TK, Wiltshire T, Andahazy M, Kay SA, Van Gelder RN, Hogenesch JB (2003) Melanopsin is required for non-image-forming photic responses in blind mice. Science 301:525-527.

Provencio I, Jiang G, DeGrip WJ, Hayes WP, Rollag MD (1998) Melanopsin: An opsin in melanophores, brain, and eye. Proc Natl Acad Sci USA 95:340-345.

Provencio I, Rodriguez IR, Jiang G, Hayes WP, Moreira EF, Rollag MD (2000) A novel human opsin in the inner retina. J Neurosci 20:600-605.

Ruby NF, Brennan TJ, Xie X, Cao V, Franken P, Heller HC, O'Hara BF (2002) Role of melanopsin in circadian responses to light. Science 298:2211-2213.

Semo M, Peirson S, Lupi D, Lucas RJ, Jeffery G, Foster RG (2003) Melanopsin retinal ganglion cells and the maintenance of circadian and papillary responses to light in aged rodless/coneless $(r d / r d c l)$ mice. Eur J Neurosci 17:1793-1801.

Szel A, Rohlich P (1992) Two cone types of rat retina detected by anti-visual pigment antibodies. Exp Eye Res 55:47-52.

Villegas-Perez MP, Lawrence JM, Vidal-Sanz M, La Vail MM, Lund RD (1998) Ganglion cell loss in RCS rat retina: a result of compression of axons by contracting intraretinal vessels linked to the pigment epithelium. J Comp Neurol 392:58-77. 